\title{
MONOMIAL REPRESENTATIONS AND GENERALIZATIONS
}

\author{
CHRISTINE BESSENRODT
}

(Received 18 April 1988)

Communicated by $\mathbf{H}$. Lausch

\begin{abstract}
We study the characteristic $p$ analogue of $M$-groups, the so-called $M_{p}$-group Generalizing this notion, we also consider the condition that the modular irreducible representations are induced from representations of dimension $<p$, or even weaker, of dimension not divisible by $p$.
\end{abstract}

1980 Mathematics subject classification (Amer. Math. Soc.) (1985 Revision): 20 C 05, 20 C 20.

\section{Introduction}

The starting point of this investigation was a paper by Okuyama [12] where the notion of $M_{p}$-groups was introduced, namely: a finite group is an $M_{p}$ group if the simple modules in characteristic $p$ are monomial. An $M$-group is an $M_{p}$-group for every prime $p$, so we have a wide class of $M_{p}$-groups by the theory of $M$-groups. We will give a criterion for a group to be an $M_{p}$ group which is related to a corresponding criterion for $M$-groups; also this motivates the definition of " $M$-blocks".

If a prime $p$ is fixed it is natural not only to look at irreducible representations which are induced from 1-dimensional representations, but also at those which are induced from representations of dimension not divisible by $p$. In the characteristic 0 situation we have, for example, that irreducible characters of a $p$-solvable group which are in a $p$-block with quaternion-free resp. modular defect group are $p^{\prime}$-induced. This condition is rather weak to

The results of this paper were announced in the Proceedings of the American Mathematical Society Summer Research Institute, Arcata (1986).

(C) 1990 Australian Mathematical Society 0263-6115/90\$A2.00+0.00 
get conditions on the group, so we will consider the stronger condition that the irreducible representations are induced from representations of dimension less than $p$. In the characteristic 0 case, then all composition factors of the group have abelian Sylow $p$-subgroups; in the characteristic $p$ case, we get that the group is $p$-solvable if we also assume that the simple modules have trivial sources. Furthermore, some results on $M_{p}$-groups and monomial representations are generalized, and it is shown how some characteristic 0 properties can be transferred to characteristic $p$.

For the following we fix some notation: $G$ is always a finite group, $p$ a prime number and $\{F, R, K\}$ is a $p$-modular splitting system for $G$, that is, $R$ is a complete discrete valuation ring with quotient field $K$ of characteristic 0 and residue field $F$ of characteristic $p$, such that $K$ and $F$ are splitting fields for $G$ and its subgroups. This last assumption will not always be needed but we put it in here for the sake of simplicity. Moreover, $R G$-modules are assumed to be $R$-free and finitely generated.

\section{Criteria for $M_{p}$-groups and $M$-blocks}

First we want to state the basic definitions precisely.

Definition 2.1. Let $A \in\{F, K\}$. An $A G$-module $V$ is called monomial if $V=W^{G}$ for some 1-dimensional $A H$-module $W$, where $H$ is a subgroup of $G$. The group $G$ is called an $M$-group if all simple $K G$-modules are monomial, and it is called an $M_{p}$-group if all simple $F G$-modules are monomial. Furthermore, $G$ is an $\bar{M}$-group resp. $\bar{M}_{p}$-group, if $G$ and all subgroups of $G$ are $M$-groups resp. $M_{p}$-groups.

Note that in general an $M$-group or $M_{p}$-group need not be an $\bar{M}$-group respectively $\bar{M}_{p}$-group; this is what makes the study of these groups difficult. For the proof of our main criterion we use the following lemma which is a characteristic $p$ version of a lemma also used to prove a corresponding result on $M$-groups (compare Dornhoff [2, 15.1], Huppert [6, V, 18.3]).

LEMMA 2.2. Let $\mathscr{H}$ be a class of finite groups such that

(1) if $G \in \mathscr{M}$, then all subgroups and factor groups of $G$ are in $\mathscr{M}$,

(2) if $G \in \mathscr{M}$ is not abelian, then there exists $A \unlhd G, A \nsubseteq Z(G)$ such that all simple $F$ A-modules are 1-dimensional.

Then all groups in $\mathscr{M}$ are $\overline{M_{p}}$-groups.

Proof. Let $G \in \mathscr{M}$. We proceed by induction on the order of $G$. Let $V$ be a simple $F G$-module. If $\operatorname{ker} V \neq 1$, then $V$ is a simple $F(G / \operatorname{ker} V)$ - 
module, and by induction and (1), $V$ is monomial. So we can assume that ker $V=1$. Abelian groups are $\bar{M}_{p}$-groups, so we can assume that $G$ is not abelian. By (2) we have $A \unlhd G, A \nsubseteq Z(G)$, such that all simple $F A$-modules are 1-dimensional. Hence $V_{A}=\bigoplus V_{i}$, where the $V_{i}$ are all 1-dimensional. As $A \nsubseteq Z(G)$ and ker $V=1$, the $V_{i}$ cannot all be isomorphic. Otherwise, take $a \in A \backslash Z(G)$, then $a$ acts as a scalar on $V$, but as $V$ is faithful, this implies $a \in Z(G)$. Contradiction. So $V=W^{G}$, where $W$ is a simple $F U$-module, $U<G$. By (1), $U \in \mathscr{M}$, so by induction, $W$ is monomial and hence $V$ is monomial. Then, clearly, $G$ is an $\overline{M_{p}}$-group.

REMARK 2.3. In the lemma above, (2) may be replaced by

(2') If $G \in \mathscr{M}$ is not abelian, then there exists $A \unlhd G, A \nsubseteq Z(G)$ such that $A$ has a normal Sylow $p$-subgroup $P$ with $A / P$ abelian.

In fact, (2) and (2') are equivalent, but we need only that $\left(2^{\prime}\right)$ implies (2). Also the following trivial extension is useful.

(2") If $G \in \mathscr{M}$ is not abelian, then $G$ is an $M_{p}$-group or there exists $A \unlhd G$, $A \nsubseteq Z(G)$ such that $A$ has a normal Sylow $p$-subgroup $P$ with $A / P$ abelian.

Before we state our criterion, which is related to a similar result on $M$ groups obtained by van der Waall [17], we recall some definitions.

Definition 2.4. (a) A class $\mathscr{F}$ of finite groups is called a formation if it satisfies

(1) $\mathscr{F}$ is closed with respect to factor groups,

(2) if $G / N$ and $G / M$ are in $\mathscr{F}$, then so is $G /(N \cap M)$.

Furthermore, a non-empty formation $\mathscr{F}$ is called saturated if $G / \phi(G) \in \mathscr{F}$ always implies $G \in \mathscr{F}$.

(b) A 2-group is called quaternion-free if the quaternion group of order 8 is not isomorphic to a homomorphic image of a subgroup of this group.

A $p$-group, for $p \neq 2$, is called modular if its subgroup lattice is modular. (This is equivalent to saying that no extra-special $p$-group of order $p^{3}$ and exponent $p$ is involved.)

THEOREM 2.5. Let $F$ be a saturated formation of groups, subgroup closed and consisting of $\overline{M_{p}}$-groups only. Let $N$ be a normal solvable subgroup of $G$ such that

(i) $G / N \in \mathscr{F}$, and

(ii) The Sylow $q$-subgroups of $N$ are quaternion-free for $q=2$, and are modular for $q \neq 2$, except possibly for $q=p$.

Then $G$ is an $\overline{M_{p}}$-group. 
Proof. For the sake of completeness we give all the details even though some parts are the same as in the characteristic 0 result (see [17]). We apply Lemma 2.2 with $\left(2^{\prime \prime}\right)$ to the class of all groups satisfying the conditions of the theorem.

First to (1). Let $H \leq G$, then $H \cap N \unlhd H$ is solvable, satisfies (ii) and $H /(H \cap N) \simeq H N / N \leq G / N$, so $H /(H \cap N) \in \mathscr{F}$. Let $M \unlhd G$ then $N M / M \unlhd G / M$ and $N M / M \simeq N /(M \cap N)$ satisfies (ii). Furthermore, $(G / M) /(N M / M) \simeq G / N M \simeq(G / N) /(N M / N) \in \mathscr{F}$.

Now to (2). If $N$ is abelian and not in $Z(G),\left(2^{\prime \prime}\right)$ is trivially satisfied. So suppose $N$ is abelian and $N \subseteq Z(G)$. As $G / N$ is an $\overline{M_{p}}$-group, it is solvable (see Section 3). Thus $G$ is solvable. By a result of Gaschütz [6, VI, 7.10b] every solvable group contains $\mathscr{F}$-covering groups, whenever $\mathscr{F}$ is saturated. By Lubeseder's theorem [6, VI, 7.25] a saturated formation of solvable groups is locally defined. Hence we can apply a result by Carter, Hawkes and Shult which says that in this situation, $G^{\mathscr{F}}$ abelian implies that $\mathscr{F}$-covering groups of $G$ are the complements of $G^{\mathscr{F}}$ in $G$ [6, VI, 7.15]. By our assumptions, $G^{\mathscr{F}} \subseteq N \subseteq Z(G)$, so $G \simeq G^{\mathscr{F}} \times D$, where $D$ is an $\mathscr{F}$-covering group of $G$. As $D$ and $G^{\mathscr{F}}$ are $\overline{M_{p}}$-groups, so is $G$.

So we can now assume that $N$ is not abelian. Suppose $N$ is not nilpotent and let $1 \neq H \unlhd N$ be minimal with $N / H$ nilpotent. Since $H$ is characteristic in $N$ it is normal in $G$. If $H$ is abelian, then we are done, because if $H \subseteq Z(G)$ then $H \subseteq Z(N)$ and $N$ is nilpotent, contradiction. So suppose $H$ is not abelian and let $A$ be the smallest non-trivial member of the derived series of $H$. Since $H$ is solvable, $A$ is abelian, and $A$ is normal in $G$ as it is characteristic in $H$. If $A \nsubseteq Z(G)$ we are done. So assume $A \subseteq Z(G)$, and hence $A \subseteq Z(N)$.

Now by $[3,8] P \cap Z(N) \cap H=1$ for all $P \in \operatorname{Syl}_{q}(N), q \neq p$. So $A$ must be a $p$-group. Now consider the next member $B$ of the derived series of $H$ (since $H$ is not abelian, such a $B$ exists). Then $A \unlhd B, B / A$ is abelian and $B$ is normal in $G$. As $B$ is not abelian, $B \nsubseteq Z(G)$. Thus we can take $B$ for the normal subgroup in $\left(2^{\prime \prime}\right)$. If $N$ is nilpotent, at least one of the Sylow subgroups is not abelian. If the Sylow $p$-subgroup is not abelian, this is a normal subgroup of $G$ satisfying $\left(2^{\prime \prime}\right)$. So suppose a Sylow $q$-subgroup for $q \neq p$ is not abelian, say $Q$. Clearly $Q \unlhd G$.

By [17, I.26 and 1.2.7] we have that there exists an abelian characteristic subgroup $L$ in $Q$ with $L \nsubseteq Z(Q)$. Hence $L$ is normal in $G$ and satisfies $L \nsubseteq Z(G)$.

Of course, to apply this theorem, we want to have interesting classes $\mathscr{F}$ which satisfy the hypothesis. For this we use the following result which again corresponds to a result on $M$-groups (see [3], [6]). 
THEOREM 2.6. Let $G$ be a finite group and suppose $G$ has a normal subgroup $N$ such that

(i) $G / N$ is $q$-supersolvable for all $q \neq p$, and

(ii) $N$ is solvable and has abelian Sylow q-subgroups for all $q \neq p$. Then $G$ is an $\overline{M_{p}}$-group.

Proof. We use Lemma 2.2. So let $\mathscr{M}$ be the class of all groups with the properties above. Clearly, $\mathscr{M}$ is closed with respect to subgroups and factor groups. Now we will prove ( $\left.2^{\prime}\right)$, so we can assume that $G \in \mathscr{M}$ is non-abelian.

Case 1. $N$ does not have a normal Sylow $p$-subgroup $P$ with $P / N$ abelian. Let $A$ be largest possible among the normal subgroups of $G$ contained in $N$ which do have a normal Sylow $p$-subgroup with abelian quotient. If $A \nsubseteq$ $Z(G)$, we are done.

So assume now that $A \subseteq Z(G)$, and thus $A \subseteq Z(N)$. Consider $N / A \unlhd G / A$. Let $B / A$ be minimal among the non-trivial normal subgroups of $G / A$ lying in $N / A$. As $N$ is solvable, $B / A$ must be abelian. Thus $B^{\prime} \subseteq A \subseteq Z(B)$, which implies that $B$ is nilpotent. As $B \unlhd N$ and $N$ has abelian Sylow $q$ subgroups for $q \neq p, B$ has a normal Sylow $p$-subgroup $P$ with $B / P$ abelian, contradicting the choice of $A$.

Case 2. $N$ has a normal Sylow $p$-subgroup $P$ with $N / P$ abelian. If $N \nsubseteq$ $Z(G),\left(2^{\prime}\right)$ is satisfied. So we can assume that $N \subseteq Z(G)$. As $G / N$ is $q$ supersolvable for all $q \neq p$, we have a series $N=G_{0} \subset G_{1} \subset \cdots \subset G_{r}=G$ where $G_{i} \unlhd G$ and $\left|G_{i+1}: G_{i}\right|$ is prime or a $p$-power. As $G$ is not abelian, there exists $i \in\{0, \ldots, r\}$ with $G_{i} \subseteq Z(G)$ and $G_{i+1} \nsubseteq Z(G)$.

If $\left|G_{i+1}: G_{i}\right|$ is prime, then $G_{i+1}$ is abelian, and we are done. So assume $\left|G_{i+1}: G_{i}\right|$ is a $p$-power. Then $G_{i+1}=G_{i} P$, where $P$ is a Sylow $p$-subgroup of $G_{i+1}$. As $G_{i} \subseteq Z(G), P \unlhd G_{i+1}$ and $G_{i+1} / P=G_{i} P / P \simeq G_{i} / G_{i} \cap P$ is abelian. Hence $G_{i+1}$ is a normal subgroup of $G$ as required in (2').

Let $\mathscr{F}_{p}$ be the class of all finite groups which are $q$-supersolvable for all $q \neq p$, and let $\mathscr{N}_{p}$ be the class of all finite groups which are $q$-nilpotent for all $q \neq p$, that is, groups which are nilpotent modulo a (normal) Sylow $p$-subgroup.

CoRollary 2.7. (a) The classes $\mathscr{F}_{p}$ and $\mathscr{N}_{p}$ are saturated subgroup-closed formations of $\overline{M_{p}}$-groups.

(b) If $G$ is a solvable group which has abelian Sylow q-subgroups for all $q \neq p$, then $G$ is an $\overline{M_{p}}$-group.

Proof. It only remains to prove that $\mathscr{F}_{p}$ and $\mathscr{N}_{p}$ are saturated formations. This follows from [6, VI, Section 8] respectively [6, VI, Section 7]. 
EXAmple 2.8. The group $G=\operatorname{SL}(2,3)$ illustrates both parts of the corollary above: $G$ is 3-supersolvable (and has abelian Sylow 3-subgroups), hence $G$ is an $\overline{M_{2}}$-group. Note that $G$ is not 2-supersolvable (and the Sylow 2subgroup is not abelian) and it is not an $M_{3}$-group.

ReMarK 2.9. (a) Combining Corollary 2.7 (a) with Theorem 2.5 gives Theorem 2.6 with abelian in (ii) replaced by quaternion-free, respectively modular. But by a result of Seitz and Wright [15] this is no more general than the original statement of 2.6 .

(b) Using results of Rigby [5] proved that Theorem 2.6 is even true if condition (i) is replaced by the more general condition

(i') $G / N$ is solvable and all $q$-chief factors of $G / N$ and its subgroups have odd degree, for all $q \neq p$.

But the resulting class of groups is not a saturated formation. Nevertheless (though not as an application of Theorem 2.5) by modifying Gow's proof we also get the result mentioned in (a) with (i) replaced by ( $\left.i^{\prime}\right)$, using again the fact that non-abelian quaternion-free, respectively modular, $p$-groups have non-central abelian characteristic subgroups. (Here the Seitz-Wright result cannot be applied!) We state this as

THEOREM 2.10. Let $G$ be a finite solvable group, and suppose $G$ has a normal subgroup $N$ such that

(i) all $q$-chief factors of $G / N$ and its subgroups have odd degree, for all $q \neq p$, and

(ii) $N$ has quaternion-free, respectively modular, Sylow q-subgroups for all $q \neq p$.

Then $G$ is an $\overline{M_{p}}$-group.

In the results above, the general translation principle from characteristic 0 to characteristic $p$ was to omit the restriction on the Sylow $p$-subgroups. Now we may go only half-way and assume that not the Sylow $p$-subgroups but the defect groups of a given $p$-block satisfy certain assumptions. Of course, we are then talking about the characteristic 0 situation, and we now define $M$-blocks.

Definition 2.11. Let $B$ be a $p$-block of $G$. Then $B$ is called an $M$-block if all irreducible characters in $B$ are monomial.

THEOREM 2.12. Let $G$ be a finite solvable group and suppose $G$ has a normal subgroup $N$ such that

(i) all chief factors of $G / N$ and its subgroups have odd degree, 
(ii) $N$ has quaternion-free, respectively modular, Sylow q-subgroups, for all $q \neq p$.

Then any p-block of $G$ with quaternion-free, respectively modular, defect group is an $M$-block.

Proof. Using Fong reduction we may assume that the defect groups of the $p$-block are Sylow $p$-subgroups, so we have to show that $G$ is an $M$-group. Since (i) and (ii) also hold for subgroups and factor groups of $G$, it suffices to prove that a faithful primitive irreducible character of $G$ is linear. Let $\chi$ be such a character. Set $H=$ Fit $N$, so $H=\prod_{i=1}^{n} Q_{i}$, where the $Q_{i}$ are the Sylow subgroups of $H$. Suppose $H$ is not abelian, so $Q_{i}$ is not abelian for some $i$. As $Q_{i}$ is quaternion-free respectively modular, it has an abelian non-central characteristic subgroup $L$. Of course, $L$ is also non-central and characteristic in $H$. So $L$ is a normal abelian subgroup of $G$, and hence must be central in $G$, as we have a faithful primitive irreducible character of $G$, a contradiction. Thus $H$ is abelian, and so $H \subseteq Z(G)$. This forces $N=H$ to be abelian and central in $G$, and therefore all chief factors of $G$ and its subgroups have odd degree. But using Rigby's result [13] it is easy to prove that such groups are $M$-groups (see Gow [5]).

REMARK 2.13. In particular, taking $N=G$ we see that $p$-blocks with quaternion-free, respectively modular, defect groups are $M$-blocks if all Sylow $q$-subgroups of $G$, for all $q \neq p$, are quaternion-free, respectively modular.

Using van der Waall's criterion for $M$-groups [17], we get similarly

THEOREM 2.14. Let $\mathscr{F}$ be a saturated subgroup-closed formation of $M$ groups. Let $G$ be a finite group and suppose $G$ has a solvable normal subgroup $N$ such that

(i) $G / N \in \mathscr{I}$,

(ii) $N$ has quaternion-free, respectively modular, Sylow q-subgroups, for all $q \neq p$.

Then any p-block of $G$ with quaternion-free, respectively, modular defect group is an $M$-block.

\section{3. $p^{\prime}$-induced representations}

In this section we want to study groups for which all irreducible representations are $p^{\prime}$-induced, that is, they are induced from representations of dimension not divisible by $p$. This is an obvious generalization of the notion 
of $M$-groups, respectively $M_{p}$-groups, and it is also a natural extension of the class of those groups whose irreducible representations are all of $p^{\prime}$-degree. Using the classification of the finite simple groups, Michler [11] proved that these last groups are characterized by the (obviously sufficient) condition that the Sylow $p$-subgroup is normal (for characteristic $p$ ), respectively normal and abelian (for characteristic 0 ). It seems to be more difficult to characterize the groups where all irreducible representations are $p^{\prime}$-induced, since this property is not inherited by normal subgroups.

But at least we have the sufficient condition that the simple $F G$-modules are all $p^{\prime}$-induced if $G$ is $p$-solvable. This was proved by Tsushima [16] and also by Gow [5]. The converse of this does not hold: for the group $A_{5}$ all simple modules in characteristic 5 are 5 -induced. Here we give a sufficient condition for the characteristic 0 case, even for a block, which generalizes a result of Gow [5].

THEOREM 3.1. Let $G$ be a p-solvable group, $B$ a p-block of $G$ with $D$ as a defect group. Suppose $G$ has a normal subgroup $N$ such that

(i) the p-chief factors of $G / N$ and all its subgroups have odd degree,

(ii) $N \cap D$ is quaternion-free, respectively modular.

Then all irreducible characters in $B$ are $p^{\prime}$-induced.

Proof. We may assume that $K$ is algebraically closed. Let $\chi$ be an irreducible character in $B$. We prove the assertion by induction on $\chi(1)$; of course, we may assume that $p$ divides $\chi(1)$. Set $H=O_{p^{\prime}}(G)$. Then $\chi_{H}=e \sum_{i} \varphi_{i}$ for some $e \in \mathbb{N}$ and irreducible characters $\varphi_{i}$ of $H$, which are all conjugate. Suppose $T=T\left(\varphi_{i}\right)<G$. Then $\chi=\varphi^{G}$ for some irreducible character $\varphi$ of $T$, and $\varphi$ lies in a block $B_{1}$ with $D$ as a defect group. Set $N_{1}=N \cap T$, so $N_{1}$ is normal in $T$ and $T / N_{1}$ satisfies (i). Moreover, $N_{1} \cap D$ is quaternion-free, respectively modular. So by induction $\varphi$ is $p^{\prime}$-induced, and hence so is $\chi$.

Thus we can now assume that $G=T\left(\varphi_{1}\right)$. In this situation, $D$ is a Sylow $p$ subgroup of $G$ and (ii) says that $N$ has quaternion-free respectively modular Sylow $p$-subgroups. We may also assume that $\chi$ is faithful and, by induction, primitive.

Now if $O_{p}(N)$ is not abelian, it has an abelian non-central characteristic subgroup $L$. As $L$ is normal in $G$, and we have an irreducible faithful primitive character of $G$, we obtain $L \subseteq Z(G)$, contradicting the fact that $L$ is non-central in $O_{p}(N)$. Thus $O_{p}(N)$ is abelian and hence $O_{p}(N) \subseteq Z(G)$. First we deal with the case that $H$ is not abelian. We are in the situation where 
$\chi_{H}=e \varphi$, with $\varphi$ an irreducible character of $H$. As $H$ is not abelian, $\varphi$ is nonlinear since $\chi$ is assumed to be faithful. Now we apply the Fong reduction (see [4, Chapter X]), so the following holds.

There exists a central extension

$$
1 \rightarrow Z \rightarrow \tilde{G} \stackrel{f}{\rightarrow} G \rightarrow 1,
$$

where $Z$ is a cyclic $p^{\prime}$-subgroup and $\widetilde{G}$ has a normal subgroup $\widetilde{H} \cong H$ such that $Z \widetilde{H}=Z \times \widetilde{H}=f^{-1}(H)$. Furthermore, if $W$ is the representation affording $\chi, V$ the representation affording $\varphi$, we have $W=\widetilde{W} \otimes \widetilde{V}$, where $\widetilde{W}$ is an irreducible $K(\widetilde{G} / \widetilde{H})$-module and $\widetilde{V}$ is a $K \widetilde{G}$-module with $f\left(\widetilde{V}_{\widetilde{H}}\right)=V$.

Thus $\operatorname{dim} \widetilde{V}=\operatorname{dim} V=\varphi(1)$ and $\operatorname{dim} \widetilde{W}=e$, and $\widetilde{G}$ is also $p$-solvable and has all the required properties. As $\varphi(1)>1, e<\chi(1)$ and by induction, $\widetilde{W}$ is $p^{\prime}$-induced. As $\varphi$ is of $p^{\prime}$-degree, this implies that $W$ and hence $\chi$ is $p^{\prime}$-induced.

Therefore, we may now assume that $H$ is abelian. As $\chi$ is assumed to be faithful, this immediately gives $H \subseteq Z(G)$. In particular, this implies $N \subseteq Z(G)$, so the $p$-chief factors of $G / Z(G)$ are of odd degree. But as $G$ is non-abelian, $O_{p}(G)$ must be non-abelian and then by [13], $G$ must have a $p$-chief factor of even degree, a contradiction.

To illustrate the above we look again at a simple example.

ExAmple 3.2. Let $G=\operatorname{SL}(2,3)$. For $p=2$, the Sylow 2-subgroup is quaternion and there exist irreducible characters of degree 2 which are not induced. For $p=3$, the Sylow 3-subgroups are even cyclic, so all irreducible characters are $3^{\prime}$-induced.

The $p^{\prime}$-induction property is closely connected to the height 0 property. First we need

Definition 3.3. Let $A \in\{R, F\}$. An indecomposable $A G$-module $V$ with $D$ as a vertex is said to be of vertex-height 0 if $\left(\operatorname{rank}_{A} V\right)_{p}=|G: D|_{p}$. (Here $n_{p}$ denotes the $p$-part of the natural number $n$.)

Proposition 3.4. (a) Let $A \in\{R, F\}$. If $V$ is an indecomposable $p^{\prime}$-induced $A G$-module, then $V$ is of vertex-height 0 .

(b) If an irreducible character $\chi$ of $G$ is $p^{\prime}$-induced, then $\chi$ is afforded by an $R G$-lattice of vertex-height 0 .

Proof. (a) Let $V=W^{G}$, where $W$ is an indecomposable $A H$-module of rank not divisible by $p, H \leq G$. Then $W$ has a Sylow $p$-subgroup $D$ of $H$ as a vertex, and a vertex $P$ of $V$ satisfies $P \leq{ }_{G} D \leq H$. As $|G: P|_{p} \leq(\operatorname{rank} V) p=$ 
$|G: H|_{p}=|G: D|_{p}$, we obtain $P={ }_{G} D$ and $(\operatorname{rank} V)_{p}=|G: P|_{p}$, and we are done.

(b) follows from (a).

COROLlary 3.5. Let $B$ be a p-block of $G$ with abelian defect group. If $\chi$ is an irreducible $p^{\prime}$-induced character in $B$, then $\chi$ is of height 0 .

Proof. By Knörr's Theorem [10], all irreducible $R G$-lattices in $B$ have the defect groups as vertices, and hence the assertion follows from Proposition 3.4(a).

As Brauer's height 0 conjecture has been proved for $p$-solvable groups by Gluck and Wolf, we also get

COROLlary 3.6. Let $G$ be a p-solvable group, $B$ a p-block of $G$ with nonabelian quaternion-free, respectively modular, defect group. Then $B$ has an irreducible $R G$-lattice with non-maximal vertex.

Proof. By Theorem 3.1, all irreducible characters in $B$ are $p^{\prime}$-induced, and hence by Proposition 3.4(a) are afforded by $R G$-lattices of vertex-height 0 . But as the defect group of $B$ is not abelian, there exists an irreducible character in $B$ which is not of height 0 . So it has an $R$-form with nonmaximal vertex.

\section{4. $r$-induced representations, $r<p$}

In this section we want to derive properties of groups all of whose irreducible representations are induced from representations of dimension less than $p$. First a general theorem which is a characteristic $p$ version of a result of Isaacs [9].

THEOREM 4.1. Let $G$ be a finite group, $\mathscr{F}$ a class of finite groups closed under isomorphisms, subgroups and extensions. Let $\widetilde{F}$ denote the smallest class of finite groups containing $\mathscr{F}$ and all p-groups which is itself closed under isomorphisms, subgroups and extensions. If all simple FG-modules are induced from simple modules over sections in $\mathscr{F}$, then $G \in \widetilde{F}$.

Proof. Let $N=G^{\tilde{g}}$ be the minimal normal subgroup of $G$ with $G / N \in \widetilde{\mathscr{F}}$. We want to show that $N=1$. If $O_{p}(G) \neq 1$, then $G / O_{p}(G) \in \widetilde{F}$ by induction, so $G \in \widetilde{F}$. Thus we may assume $O_{p}(G)=1$. 
If $N \neq 1$, then there exists a simple $F G$-module $S$ with $N \nsubseteq \operatorname{ker} S$. If $\operatorname{ker} S \neq 1$, then $G / \operatorname{ker} S \in \widetilde{F}$ by induction and hence $N \subseteq \operatorname{ker} S$, a contradiction. Thus ker $S=1$. Now choose $S$ to be a faithful simple $F G$-module of minimal dimension. By hypothesis, $S=U^{G}$, where $U$ is a simple $F H$ module, $H<G$, and $H / \operatorname{ker} U \in \mathscr{F}$.

We consider the module $V=\left(F_{H}\right)^{G}$. Clearly $\operatorname{dim} V \leq \operatorname{dim} S$. As $F_{G}$ is a composition factor of $V$, every composition factor of $V$ has dimension $<\operatorname{dim} S$. So by the choice of $S, N$ is in the kernel of every composition factor of $V$.

Since $G / O^{p}(N)$ is an extension of $G / N \in \widetilde{F}$ by the $p$-group $N / O^{p}(N)$, we have $G / O^{p}(N) \in \widetilde{F}$ and hence $N=O^{p}(N)$ is generated by $p$-regular elements. Thus $N \subseteq \operatorname{ker} V \subseteq H$. Now $H / N \in \widetilde{\mathscr{F}}$, so $M=H^{\tilde{F}} \subseteq N$. But $N / M \leq H / M \in \widetilde{F}$ implies $N^{\widetilde{F}}=N \subseteq M$, and thus $N=M$. As $H / \operatorname{ker} U \in \mathscr{F} \subseteq \mathscr{F}$, we obtain $N=M \subseteq \operatorname{ker} U$ and hence $N \subseteq \operatorname{ker} S=1$.

COROLLARY 4.2. If all simple FG-modules are induced from solvable sections, then $G$ is solvable. In particular, $M_{p}$-groups are solvable.

Remark 4.3. Dornhoff [3] proved the corollary above in the characteristic 0 case. In a remark he states a more general result but not quite correctly.

For $M_{p}$-groups there is an even more precise result, which was obtained independently by Gow [5] and Okuyama [12]. For completeness we include here a slightly more general version of this.

Proposition 4.4. Let $G$ be a finite group, $d$ an integer such that all simple $F G$-modules of dimension $\leq d$ are monomial. Let $1=d_{1}<d_{2}<\cdots<d_{k} \leq d$ be the dimensions occurring. If $V$ is a simple $F G$-module of dimension $d_{i}$, then $G^{p(i)} \subseteq \operatorname{ker} V$. (Here we set $G^{p(0)}=G, G^{p(i)}=O^{p}\left[G^{p(i-1)}, G^{p(i-1)}\right]$.)

Proof. For $i=1$ the assertion is clear. We proceed by induction. Let $i>1$. As $d_{i} \leq d, V$ is monomial, say $V=W^{G}$, where $W$ is a 1-dimensional $F H$-module, $H<G$. We consider the module $\left(F_{H}\right)^{G}$. If $S$ is any composition factor of $\left(F_{H}\right)^{G}$, then $\operatorname{dim} S<|G: H|=\operatorname{dim} V$, and by induction $G^{p(i-1)} \subseteq$ $\operatorname{ker} S$. As $G^{p(i-1)}$ is generated by $p$-regular elements, $G^{p(i-1)} \subseteq \operatorname{ker}\left(F_{H}\right)^{G} \subseteq H$. Therefore, $G^{p(i)} \subseteq O^{p}\left(H^{\prime}\right) \subseteq \operatorname{ker} W$. As $G^{p(i)}$ is normal in $G$, this implies $G^{p(i)} \subseteq \operatorname{ker} V$.

Now we want to use Isaacs' Theorem [9], respectively our Theorem 4.1, to arrive at the necessary conditions announced earlier. First we note 
Lemma 4.5. Let $G$ be a finite group, $A \in\{R, F\}$, and $V$ an indecomposable AG-module.

(i) If $V=L^{G}$, where $L$ is an AH-module of $p^{\prime}$-rank, and $\mathrm{vx} L \subseteq \operatorname{ker} L$, then $V$ is induced from a $p^{\prime}$-section.

(ii) If $V$ has trivial source and $V=L^{G}$, where $L$ is an $A H$-module of rank less than $p$, then $V$ is induced from a $p^{\prime}$-section.

Proof. (i) is obvious. For (ii), we only have to show vx $L \subseteq \operatorname{ker} L$. By Proposition 3.4(b), a Sylow $p$-subgroup $D$ of $H$ is a vertex of $V$ and $L$. As $V$ has trivial source, $L_{D} \mid\left(A_{D}^{G}\right)_{D}=\bigoplus_{D \backslash G / D} A_{D^{x} \cap D}^{D}$ and $\operatorname{rank} L<p$ implies $L_{D} \cong(\operatorname{rank} L) A_{D}$, so $D \subseteq \operatorname{ker} L$.

Proposition 4.6. Let $G$ be a finite group of all of whose irreducible characters are induced from characters of degree less than $p$. Then all composition factors of $G$ have abelian Sylow p-subgroups.

Proof. Let $\mathscr{F}$ be the class of all finite groups whose composition factors all have abelian Sylow $p$-subgroups. Clearly, $\mathscr{F}$ is closed with respect to subgroups, isomorphisms and extensions. So it suffices to prove that all irreducible characters are induced from sections in $\mathscr{F}$ (by [9]). So let $\chi$ be an irreducible character of $G$. By hypothesis, $\chi=\varphi^{G}$ for some irreducible character $\varphi$ of $H \leq G$ with $\varphi(1)<p$. Let $D$ be a Sylow $p$-subgroup of $H$, so $\varphi_{D}$ is a sum of linear characters and $D^{\prime} \subseteq \operatorname{ker} \varphi$. Thus $H / \operatorname{ker} \varphi$ has abelian Sylow $p$-subgroups.

Note that for example all irreducible characters of $A_{5}$ are induced from characters of degree less than 5 .

For the characteristic $p$ result, we have to put in the extra assumption that the simple modules have trivial source, which is trivially satisfied if the simple modules are monomial.

Proposition 4.7. Let $G$ be a finite group.

(i) If all simple $F G$-modules are induced from $p^{\prime}$-dimensional modules with $\mathrm{vx} \subseteq \mathrm{ker}$, then $G$ is $p$-solvable.

(ii) If all simple $F G$-modules have trivial sources and they are induced from modules of dimension less than $p$, then $G$ is p-solvable.

Proof. This follows from Theorem 4.1 by using Lemma 4.5(i) resp. (ii).

REMARK 4.8. (a) "Trivial source" is really an extra condition, even in the solvable case. Take again $G=\operatorname{SL}(2,3), p=3$. The simple $F G$-modules $I$, 
2, 3 are all induced from modules of dimension less than 3 , but 2 does not have trivial source.

(b) For the lattice version of Proposition 4.7(ii), the hypothesis "trivial source" is too restrictive: if every irreducible character has an $R$-form of rank less than $p$ and has trivial source, then $G$ is already a $p^{\prime}$-group (use Lemma 4.5 and Isaacs' Theorem [9]).

(c) Assertion (ii) is not true without the assumption on the sources: take again $G=A_{5}, p=5$, here the simple modules are induced from modules of dimension 1 and 3.

The existence of a faithful module of dimension less than $p$, together with the "trivial source" property only for simple modules of small dimension, has strong implications on the group.

Proposition 4.9. Let $G$ be a finite group, all of whose simple FG-modules of dimension less than $p$ have trivial sources. If there exists an FG-module $V$ with $\operatorname{dim} V<p$ and $\operatorname{ker} V$ a p-group, then $G$ has a normal Sylow p-subgroup.

Proof. By induction on the order of $G$, we may assume that ker $V=1$. Now take a faithful $F G$-module $V$ of minimal dimension.

If $V$ is not simple, let $V_{i}, 1 \leq i \leq n$, denote the composition factors of $V$. Since $\operatorname{dim} V_{i}<\operatorname{dim} V$, we have $Y_{i}=\operatorname{ker} V_{i} \neq 1$ for all $i$. By induction, $G / Y_{i}$ has a normal Sylow $p$-subgroup, and hence so has $\prod_{i} G / Y_{i}$. Now $N=\bigcap_{i} Y_{i}$ is a $p$-group, since otherwise $N$ contains a $p$-regular element $x \neq 1$ and this would lie in $\operatorname{ker} V=1$, a contradiction. As $G / N \hookrightarrow \prod_{i} G / Y_{i}, G / N$ has a normal Sylow $p$-subgroup, and hence so has $G$. Thus we can now assume that $V$ is simple. But then Lemma 4.5 (ii) immediately implies that $G$ is a $p^{\prime}$-group.

As a corollary, we get a characteristic $p$ version of a result on $M$-groups (see Seitz [15]).

COROLlary 4.10. Let $G$ be a finite group, whose simple FG-modules of dimension less than $p$ are monomial. If there exists an $F G$-module $V$ with $\operatorname{dim} V<p$ and $\operatorname{ker} V$ a $p$-group, then $G$ has a normal Sylow p-subgroup and is solvable.

In particular, an $M_{p}$-group with a faithful FG-module of dimension less than $p$ has a normal Sylow p-subgroup.

Proof. To see that $G$ is solvable, note that we may assume that $V$ is simple, by using induction and the same arguments as in the proof of Proposition 4.9. Then the assertion follows from Proposition 4.4. 
EXAMPLE 4.11. The groups $\operatorname{SL}(2,3)$ and $\operatorname{GL}(2,3)$ have faithful modules of dimension 2 (for $p=3$ ), but are not $M_{3}$-groups. Their Sylow 3-subgroups are not normal.

For $M$-groups it is well-known that normal Hall subgroups are again $M$ groups [3]. The same holds for $M_{p}$-groups (see [5]), but in fact we have

THEOREM 4.12. Let $G$ be a p-solvable group, $N$ a normal Hall subgroup of $G$. Let $\mathscr{D}$ be any set of divisors of the order of $N$. If all simple $F G$-modules are induced from modules of dimension in $\mathscr{D}$, then the same holds for the simple $F N$-modules.

Proof. Let $V$ be a simple $F N$-module. By the Mackey decomposition, $\left(V^{G}\right)_{N}=|T: N| \bigoplus_{x \in G / T} V^{x}$, where $T=T_{G}(V)$. By [7, VII, 9.7] there exists a simple $F T$-module $W$ with $V \simeq W_{N}$. Then $W^{G}$ is a simple $F G$-module, so $W^{G}=U^{G}$, where $U$ is a simple $F H$-module, $H \leq G$, with $s=\operatorname{dim} U$ in $\mathscr{D}$. Then

$$
s|G: H|=|G: T| \operatorname{dim} W=|G: T| \operatorname{dim} V .
$$

Set $\pi=\{q$ prime, $q \nmid|N|\}$ and denote by $n_{\pi}$ the $\pi$-part of the natural number $n$. Then we get

$$
|G|_{\pi}=|G: N|, \quad|G: H|_{\pi}=|G: T|,
$$

as $s|| N \mid$ and $N$ is $p$-solvable and hence $\operatorname{dim} V|| N \mid$. Thus $|H|_{\pi}=|T: N|$ and so $|H: H \cap N|=|T: N|$. Set $\widetilde{H}=H \cap N$ and $\widetilde{U}=U_{\widetilde{H}}$. Using the Nakayama relations we obtain

$$
\operatorname{dim} \operatorname{Hom}_{\widetilde{H}}\left(\widetilde{U},\left(U^{G}\right)_{\widetilde{H}}\right)=\operatorname{dim} \operatorname{Hom}_{G}\left(\widetilde{U}^{G}, U^{G}\right)=\operatorname{dim} \operatorname{Hom}_{G}\left(\left(\widetilde{U}^{H}\right)^{G}, U^{G}\right) .
$$

Now this dimension is positive, since $\operatorname{dim} \operatorname{Hom}_{H}\left(\widetilde{U}^{H}, U\right)=\operatorname{dim} \operatorname{Hom}_{\widetilde{H}}(\widetilde{U}, \widetilde{U})$ is positive. As we have

$$
\left(U^{G}\right)_{N}=\left(W^{G}\right)_{N} \cong \bigoplus_{G / T} W^{x}{ }_{N} \cong \bigoplus_{G / T} V^{x},
$$

there must be some $x \in G$ with

$$
0 \neq \operatorname{dim} \operatorname{Hom}_{\widetilde{H}}\left(\widetilde{U}, V^{x} \widetilde{H}^{\prime}\right)=\operatorname{dim} \operatorname{Hom}_{N}\left(\widetilde{U}^{N}, V^{x}\right) .
$$

But

$$
\begin{aligned}
\operatorname{dim} \widetilde{U}^{N} & =s|N: \widetilde{H}|=s \frac{|G: \widetilde{H}|}{|G: N|}=s \frac{|G: H||H: \widetilde{H}|}{|G: N|} \\
& =s \frac{|G: H||T: N|}{|G: T||T: N|}=s \frac{|G: H|}{|G: T|}=\operatorname{dim} V
\end{aligned}
$$

and $V^{x}$ is simple, hence $\widetilde{U}^{N} \cong V^{x}$ and thus also $V$ is induced from a module of dimension $s$ in $\mathscr{D}$. 
CoROLLARY 4.13. Normal Hall subgroups of $M_{p}$-groups are $M_{p}$-groups.

Proof. $M_{p}$-groups are $p$-solvable, so apply Theorem 4.12 with $\mathscr{D}=\{1\}$.

RemarK 4.14. One can modify Dornhoff's proof for $M$-groups [3] to obtain a statement analogous to Theorem 4.12 also in the characteristic 0 case.

\section{Green correspondence}

Generalizing a result of Okuyama [12] on monomial simple $F G$-modules, we want to investigate how the modules studied above behave with respect to Green correspondence. For this we fix some more notation.

Let $D$ be a $p$-subgroup of $G, N=N_{G}(D)$ and set

$$
\begin{aligned}
& \mathscr{X}=\left\{Y \leq G \mid Y \subseteq D \cap D^{x} \text { for some } x \in G \backslash N\right\}, \\
& \mathscr{Y}=\left\{Y \leq G \mid Y \subseteq N \cap D^{x} \text { for some } x \in G \backslash N\right\}, \\
& \mathscr{Z}=\{Y \leq N \mid Y \cap D<D\} .
\end{aligned}
$$

Furthermore, let $f$ and $g$ denote the Green correspondences with respect to $D$ (see for example Feit [4]), $A \in\{R, F\}$.

In the paper mentioned above Okuyama proved the following. Let $S$ be a simple $F G$-module with vertex $D$. Then $S$ has trivial source if and only if $f(S)$ is simple. Furthermore, if $S$ is monomial then so is $f(S)$.

Now it is natural to ask for a characterization of simple $F G$-modules $S$ with monomial Green correspondents $f(S)$, respectively simple $F N$-modules $U$ with monomial Green correspondents $g(U)$. It turns out that we can extend Okuyama's result to $r$-induced modules, where $r<p$. In fact we only need an easy property of modules of small dimension, which leads to the following

Definition 5.1. An $A G$-module $L$ is called $D$-regular if all indecomposable summands of $L_{D}$ have vertex $D$. If, in addition, $L$ is indecomposable and has $D$ as a vertex, $L$ is called vertex-regular. If $X$ is any $p$-group, $L$ is called $X$-isoregular if $L$ is $Y$-regular for all $Y \leq G$ isomorphic to $X$.

REMARK 5.2. Note that a module of rank $r<p$ is $X$-isoregular for any $p$-group $X$. So the statement of the next theorem looks somewhat nicer for this particular case.

THEOREM 5.3. Let $U$ be an indecomposable $A N$-module with vertex $D$, $r \in \mathbb{N}$ not divisible by $p$. Then the following are equivalent. 
(i) $U$ is r-induced.

(ii) There exists an AG-module $V$ which is induced from a D-isoregular module of rank $r$, such that $V=g(U) \oplus \mathscr{X}$-projectives.

(iii) There exists an AG-module $V$ which is induced from a D-isoregular module of rank $r$, such that $V_{N}=U \oplus \mathscr{Y}$-projectives.

(iv) There exists an AG-module $V$ which is induced from a $D$-isoregular module of rank $r$, such that $V_{N}=U \oplus \mathscr{Z}$-projectives.

Proof. (i) implies (ii). Say $U=L^{N}$, where $L$ is an $A H$-module of rank $r$, $H \leq N$. Then $L$ is $D$-isoregular (since $D$ is the Sylow $p$-subgroup of $H$ ) and $L^{G}=\left(L^{N}\right)^{G}=U^{G}=g(U) \oplus \mathscr{Z}$-projectives. Set $V=L^{G}$.

(ii) implies (iii) follows directly from the properties of the Green correspondence.

(iii) implies (iv) is trivial.

(iv) implies (i). Say $V=L^{G}$, where $L$ is a $D$-isoregular $A H$-module of rank $r, H \leq G$. Decompose $L^{G}=\bigoplus_{i} V_{i}$ with indecomposable $A G$-modules $V_{i}$. By assumption, there exists $i$ such that $\left.V_{i}\right|_{N}=U \oplus \mathscr{Z}$-projectives. Then $D$ must be a vertex of $V_{i}$ and $V_{i}=g(U)$ by [1]. As $V_{i} \mid L^{G}$, we have $D \leq H^{z}$ for a suitable $z \in G$. Now $\left(L^{G}\right)_{N}=\bigoplus_{H \backslash G / N}\left(L_{H^{x} \cap N}^{x}\right)^{N}$ and $\left(\left(L_{H^{z} \cap N}^{z}\right)^{N}\right)_{D}=$ $\bigoplus_{N / H^{z} \cap N} L_{D}^{z x}$, which implies that $\left(L_{H^{2} \cap N}^{z}\right)^{N}$ is $\mathscr{Z}$-projective-free, as $L$ is $D$ isoregular. Since $U$ is indecomposable, we must have $U \simeq\left(L_{H^{z} \cap N}^{z}\right)^{N}$, so $U$ is $r$-induced.

COROllary 5.4. Let $V$ be an indecomposable AG-module with vertex $D$. If $V$ is $r$-induced for $r<p$, then so is $f(V)$.

For the converse, we can derive a somewhat technical condition from

THeOREM 5.5. Let $V$ be an indecomposable $A G$-module with vertex $D$, $r \in \mathbf{N}$ not divisible by $p$. Then the following are equivalent.

(i) $V$ is induced from a $D$-isoregular module of rank $r$.

(ii) There exists $H \leq G$ and a $D$-isoregular $A H$-module $L$ of rank $r$ with $f(V)=\left(L_{H \cap N}\right)^{N}$, and $L^{G}$ is indecomposable.

Proof. (i) implies (ii) follows from the proof of 5.3(iv) implies (i).

(ii) implies (i). $f(V)=\left(L_{H \cap N}\right)^{N} \mid\left(L^{G}\right)_{N}$, and hence by [1] we must have that $D$ is a vertex for $L^{G}$ and $L^{G}=g f(V)=V$. 


\section{Acknowledgment}

The author would like to thank the Deutsche Forschungsgemeinschaft for support during part of the time when this article was written. Thanks go also to $\mathrm{R}$. Gow for sending parts of his thesis.

\section{References}

[1] D. W. Burry and J. F. Carlson, 'Restrictions of modules to local subgroups', Proc. Amer. Math. Soc. 84 (1982) 181-184.

[2] L. Dornhoff, Group representation theory, Part A (Marcel Dekker, New York, 1971).

[3] L. Dornhoff, ' $M$-groups and 2-groups' Math. Z. 100 (1967) 226-256.

[4] W. Feit, The representation theory of finite groups (North-Holland, 1982).

[5] R. Gow, The p-subgroups of classical groups and some topics in the representation theory of soluble groups (Ph.D. Thesis, Liverpool, 1973).

[6] B. Huppert, Endliche Gruppen I (Springer, 1967).

[7] B. Huppert and N. Blackburn, Finite groups II (Springer, 1982).

[8] B. Huppert, 'Gruppen mit modularer Sylowgruppe,' Math. Z. 75 (1961), 140-153.

[9] I. M. Isaacs, 'Generalizations of Taketa's theorem on the solvability of $M$-groups', Proc. Amer. Math. Soc. 91 (1984), 192-194.

[10] R. Knörr, 'On the vertices of irreducible modules', Ann. of Math. 110 (1979), 478-499.

[11] G. O. Michler, Brauer's conjectures and the classification of finite simple groups, (Springer Lecture Notes in Math. 1178, 1986, pp. 129-142).

[12] T. Okuyama, 'Module correspondence in finite groups,' Hokkaido Math. J. 10 (1981), 299318.

[13] J. F. Rigby, 'Primitive linear groups containing a normal nilpotent subgroup larger than the centre of the group,' J. London Math. Soc. 35 (1960), 389-400.

[14] G. M. Seitz and C. R. B. Wright, 'On finite groups whose Sylow subgroups are modular or quaternion-free,' J. Algebra 13 (1969), 374-381.

[15] G. M. Seitz, 'M-groups and the supersolvable residual,' Math. $Z$. 110 (1969), 101-122.

[16] Y. Tsushima, 'On the second reduction theorem of P. Fong,' Kumamoto J. Sci. (Math.) 13 (1978), 1-5.

[17] R. W. van der Waall, 'On monomial groups,' J. Reine Angew. Math. 264 (1973), 103-134.

Fachbereich 11, Mathematik

Lotharstrasse 65

D-4100 Duisburg

Bundesrepublik Deutschland 